\title{
Surgical Removal of Multiple Gastric Foreign Bodies. Case Report
}

\author{
Pyong Wha Choi* \\ Department of Surgery, Inje University College of Medicine, Ilsan Paik Hospital, 170, \\ Juhwa-ro, Ilsanseo-gu, Goyang-si, Gyeonggi-do, 10380, Korea \\ *Corresponding author: choipeace1130@gmail.com
}

Received November 17, 2018; Revised December 28, 2018; Accepted January 21, 2019

\begin{abstract}
In the adult population, psychiatric disorders and mental retardation are risk factors for foreign body ingestion. Most cases of foreign body ingestion are managed by close observation or endoscopic intervention. However, surgical intervention is mandatory in some cases. Here, we present the case of an unusually huge amount of gastric foreign bodies, which were removed by surgical intervention. A 54-year-old male patient presented with epigastric pain. He was born with mental retardation and had a 10-year history of anxiety disorder. Before the presentation, a colonic foreign body (coin) was identified 5 months ago, and the foreign body had been passed through the colon without morbidity. Plain radiography showed several foreign bodies occupying the whole stomach, and computed tomography showed multiple oval-shaped radiopaque foreign bodies in the stomach without complications. Although gastroscopy was attempted to extract these foreign bodies, it was impossible to extract all of them. Thus, surgery was performed. The foreign bodies (pebbles and coins) were extracted by surgery, and plain radiography was performed to confirm that there were no residual foreign bodies. The postoperative course was uneventful. Three months after the surgery, a radiopaque round foreign body was detected, and it was resolved without any intervention. Thereafter, the patient course was uneventful during the 1-year follow-up. Although a case with such a large number of gastric foreign bodies is extremely rare in humans, it is important to have a close follow-up after the removal of foreign bodies in patients with psychiatric disorders.
\end{abstract}

Keywords: foreign body, pebbles, stomach, psychiatric patient, surgery

Cite This Article: Pyong Wha Choi, "Surgical Removal of Multiple Gastric Foreign Bodies. Case Report." Journal Name, vol. 7, no. 1 (2019): 1-4. doi: 10.12691/ajmcr-7-1-1.

\section{Introduction}

Physicians commonly encounter patients with foreign body ingestion in clinical practice. Most cases comprise pediatric patients. In the adult population, psychiatric patients or patients with mental retardation are vulnerable to foreign body ingestion. [1] Most gastric foreign bodies are managed by observation or endoscopic intervention. However, some patients need surgery to extract foreign bodies or treat complications. [2,3] Here, we present an extremely unusual case with a large number of gastric foreign bodies, which were extracted by surgical intervention.

\section{Case Report}

A 54-year-old male patient was referred to the emergency department after presenting with epigastric pain. The pain had started approximately one week prior to presentation and had intensified gradually over time. the patient, who was born with mental retardation and had a 10-year history of anxiety disorder, was previously treated at the psychological department. Before presentation, a colonic foreign body (coin) was identified at the time of admission to the psychologic department (5 months previously). At that time, the foreign body had passed through the colon without morbidity (Figure 1).

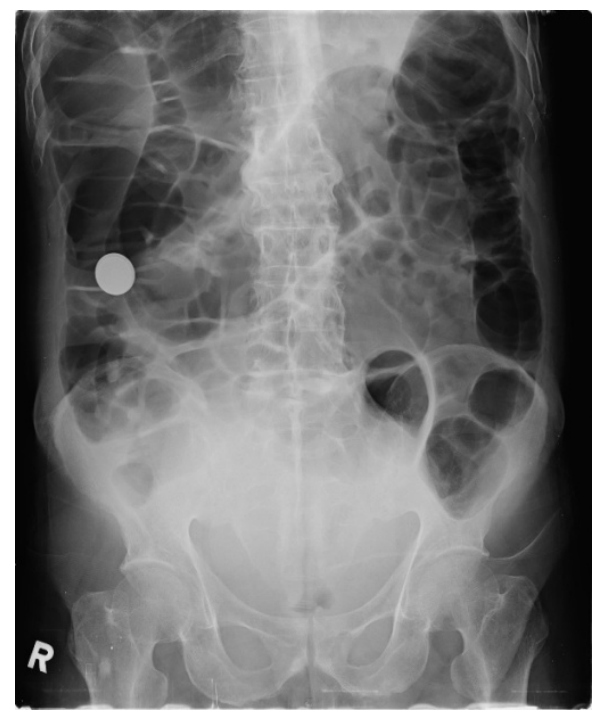

Figure 1. Plain radiography shows a round radiopaque foreign body in the ascending colon 
The vital signs at admission were body temperature, $37.0^{\circ} \mathrm{C}$; blood pressure, $115 / 88 \mathrm{mmHg}$; heart rate, 82 beats/minute and respiratory rate, 18 breaths/minute. The abdomen was slightly distended and there were no signs of peritoneal irritation. However, something resembling small stones could be felt at the epigastrium. Although the laboratory results were unremarkable, abdominal radiography showed a huge amount of foreign bodies occupying the whole stomach (Figure 2).

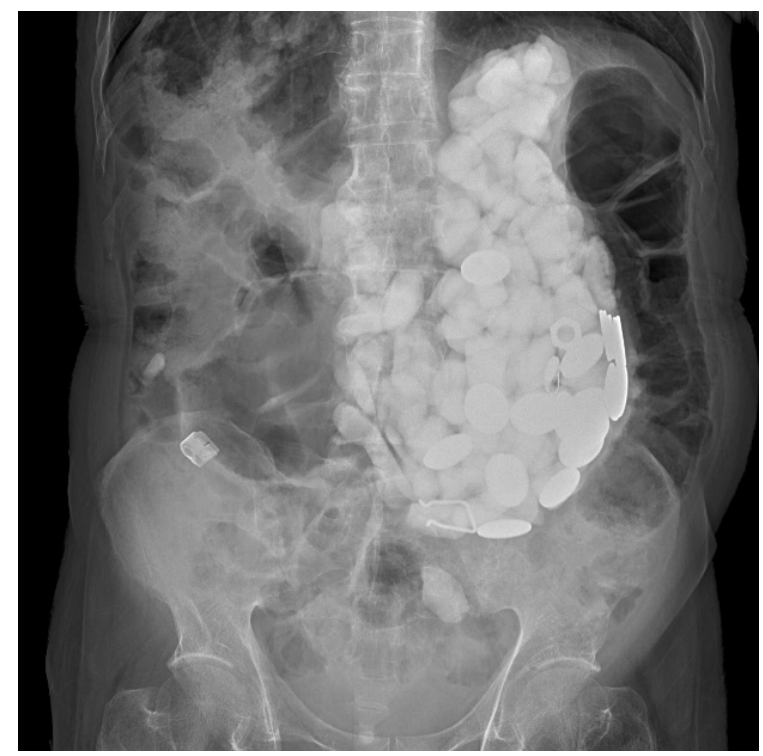

Figure 2. Plain radiography shows multiple radiopaque foreign bodies in the whole stomach and a foreign body in the descending colon

Abdominal computed tomography showed multiple oval-shaped radiopaque foreign bodies in the stomach without complications (Figure 3).

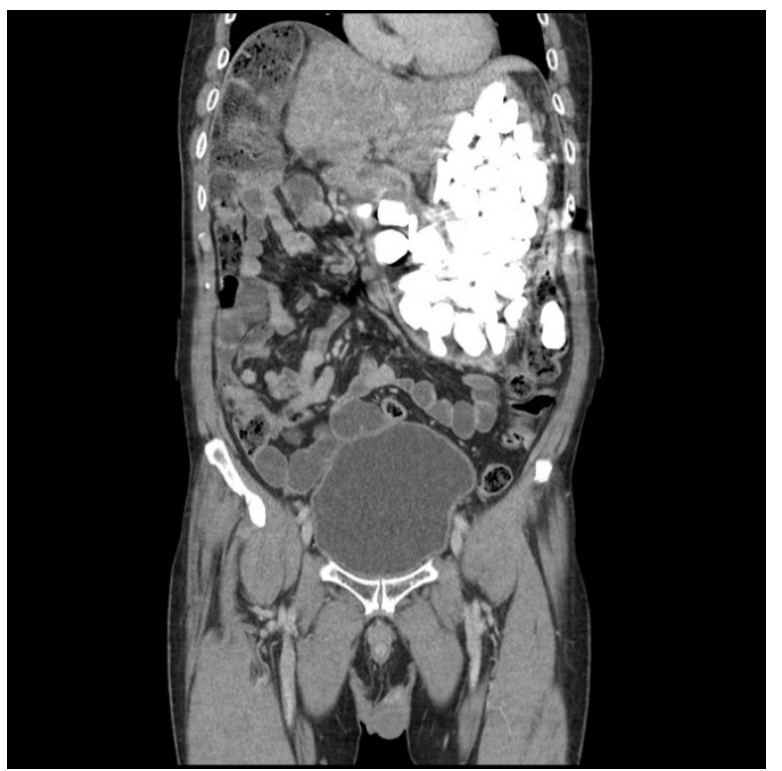

Figure 3. Abdominopelvic computed tomography shows multiple foreign bodies in the stomach without evidence of complications

It was discovered that, in the past, the patient had a habit of eating foreign bodies such as pebbles and coins whenever he felt anxious despite taking anti-anxiety medication. We attempted to extract the foreign bodies by gastroscopy, but failed in our attempt because of the large number of foreign bodies present (Figure 4).

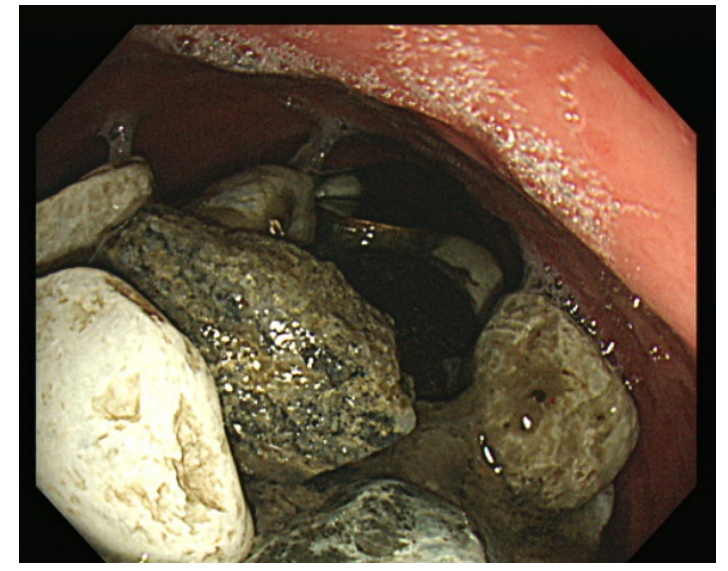

Figure 4. Gastroscopy shows multiple impacted pebbles and coins in the stomach

Thus, on the 2nd hospital day, laparotomy was performed. During the operation, gastrotomy was performed on the dependent portion of the stomach body and the foreign bodies were extracted one by one. The extracted foreign bodies composed of pebbles, coins, and bottle caps, weighing approximately $2 \mathrm{~kg}$ (Figure 5).

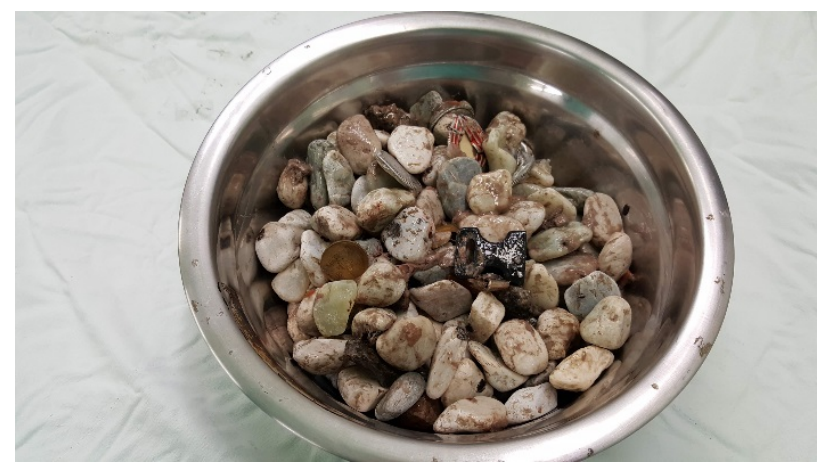

Figure 5. Extracted foreign bodies are composed of pebbles, coins and bottle caps

After the extraction of the foreign bodies, simple postoperative radiographic examination was performed to confirm residual gastric foreign bodies and primary repair was performed. The postoperative course was uneventful, and the patient was discharged on the ninth postoperative day. No evidence of abdominal foreign bodies was indicated by the findings of a simple radiographic examination performed during follow-up at 1 month after surgery. However, after 3 months, a radiopaque round foreign body was detected, and it was resolved without any intervention. Thereafter, the patients' course was uneventful during the 1 year follow-up.

\section{Discussion}

The ingestion of a foreign body commonly occurs in clinical practice. Pediatric patients represent a high-risk population as they may accidentally swallow various foreign bodies such as coins and toys. In adult patient groups, common ingested foreign bodies are fish bones, bones, and dentures. [1,2,3] The ingestion of foreign bodies can occur accidently or intentionally, depending on the risk group of the patient. Whereas mentally retarded patients swallow foreign body accidentally, patients with 
psychiatric disorders may swallow foreign body on purpose. [1] In the present case, although the patient was mentally retarded and recognized that eating pebbles and coins are dangerous, he used to eat foreign bodies to relieve anxiety symptoms. Although some ingested gastric foreign bodies have been reported, innumerable impacted gastric foreign bodies in humans have not been reported in the English literature. [4]

The patients may present with diverse symptoms depending on the location of the ingested foreign body. In cases of esophageal foreign bodies, foreign body sensation, dysphasia, and swallowing difficulty may be present. However, once the foreign bodies pass through the esophagus, symptoms associated with the foreign body is rare. [5] In the present case, even though the patient did not complain of vomiting, the large number of pebbles and coins in his stomach caused him abdominal pain.

In general, the diagnosis of foreign body ingestion is made based on looking at the patient's history. For mentally healthy adult patients, a history of ingestion may be one of the most important clues. However, in mentally impaired patients, looking at the patient's history may not be enough for to make a diagnosis. [5,6] Although physical examination is inevitable for the patients, palpation of the gastric foreign body is difficult unless large sized or large amounts of gastric foreign bodies are present, such as in our case. Thus, imaging studies are necessary in patients with suspicion of foreign body ingestion. Plain radiography is recommended to evaluate the presence, location, size, configuration and number of the foreign bodies, even though some of the foreign bodies such as fish bone, wood and plastic objects may not be detected. [7,8] In these cases, CT may be the imaging modality of choice. CT not only enables the detection of radiolucent foreign bodies, but also complications associated with foreign bodies such as perforation and abscess formation. [9] In this case, pebbles and coins in the stomach could be readily detected by plain radiography. Therefore, abdominal CT may be dispensable in this case. However, we could confirm the absence of complications and foreign bodies in the other small and large bowel. Although the usefulness of CT as a screening tool for patients with ingested foreign body has not been well established, CT may be necessary for the evaluation of the presence of complications, as some complications such as retroperitoneal perforation without free air may not be detected through plain radiography alone.

The management of ingested foreign bodies depends on the presence of complications, the size and nature of the foreign body and anatomic location. [7,8] Over $80 \%$ of ingested foreign bodies pass though the gastrointestinal tract without morbidity. [6] Particularly, close observation may be the treatment of choice for blunt ingested foreign bodies less than $2.5 \mathrm{~cm}$ in diameter and $6 \mathrm{~cm}$ in length, as the pylorus and duodenal $1^{\text {st }}-3^{\text {rd }}$ part are the main lodging points. However, it is recommended to perform endoscopic intervention when the gastric foreign body remains in the stomach for 3-4 weeks. [10,11] Endoscopy not only enables the confirmation of the shape and nature of the foreign body, but also provides therapeutic options. Even though the success rate is more than $90 \%$ and less than $1 \%$ of patients with ingested foreign bodies require surgical intervention, when endoscopy fails, or complications occur, surgery is indicated. [10,11] Delayed presentation, sharp objects, psychiatric disorders and multiple objects have been reported as risk factors for complication associated with ingested foreign bodies. [3] Although delayed presentation, psychiatric disorder and multiple objects correspond to risk factors in this case, complications did not ensue. However, surgery was inevitable as there were too many foreign bodies to extract using endoscopy.

After removing the foreign bodies, it is necessary to ascertain whether the patient will swallow foreign bodies again, particularly in patients with psychiatric disorders. Regular serial plain radiography may be the optimal follow-up tool. In this case, after the first foreign body ingestion, we could not check plain radiography due to follow-up loss for 5 months and the patient did not visit psychological department. If we could have checked serial plain radiography and the patient had received psychological treatment for anxiety, he could have been managed through close observation or endoscopic intervention, avoiding surgery. Therefore, close follow-up and treatment for underlying diseases are necessary for patients with psychiatric disorders, after the removal of foreign bodies.

\section{Conclusions}

Cases with a large number of gastric foreign bodies are extremely rare in humans. However, such foreign body ingestion may occur in patients with psychiatric disorders for secondary gain or other purposes. When managing such patients, it is important not only to perform close follow-up but also to treat underlying psychiatric disorders after the removal of foreign bodies.

\section{Acknowledgements}

The author has no sources of funding to report.

\section{Competing Interests}

\author{
No competing interests
}

\section{References}

[1] Pavlidis, T.E., Marakis, G.N., Triantafyllou, A., Psarras, K., Kontoulis, T.M. and Sakantamis, A.K, "Management of ingested foreign bodies. How justifiable is a waiting policy?", Surg Laparosc Endosc Percutan Tech, 18(3). 286-287. June 2008.

[2] Sugawa, C., Ono. H., Taleb, M. and Lucas, C.E, "Endoscopic management of foreign bodies in the upper gastrointestinal tract: A review", World J Gastrointest Endosc, 6(10). 475-481. October 2014.

[3] Lin, H.H., Lee, S.C., Chu, H.C., Chang, W.K., Chao, Y.C. and Hsieh, T.Y, "Emergency endoscopic management of dietary foreign bodies in the esophagus", Am J Emerg Med, 25(6). 662665. July 2007.

[4] Chang, W.J. and Chiu, W.Y, "Gastric foreign body: a comb", Clin Case Rep, 5(6). 1036-1037. April 2017.

[5] Geraci, G., Sciume', C., Di Carlo, G., Picciurro, A. and Modica, G, "Retrospective analysis of management of ingested foreign bodies and food impactions in emergency endoscopic setting in adults", BMC Emerg Med, 16(1). 1-5. November 2016. 
[6] Erbil, B., Karaca, M.A., Aslaner, M.A., Ibrahimov, Z., Kunt, M.M., Akpinar, E. and Özmen, M.M, "Emergency admissions due to swallowed foreign bodies in adults", World J Gastroenterol, 19(38). 6447-6452. October. 2013.

[7] Lee, J.H., Kim, H.C., Yang, D.M., Kim, S.W., Jin, W., Park, S.J. and Kim, H.J, "What is the role of plain radiography in patients with foreign bodies in the gastrointestinal tract?", Clin Imaging, 36(5). 447-454. September-October 2012.

[8] Ayantunde, A.A. and Oke, T, "A review of gastrointestinal foreign bodies”, Int J Clin Pract, 60(6). 735-739. June 2006.
[9] Coulier, B., Tancredi, M.H. and Ramboux, A, "Spiral CT and multidetector-row CT diagnosis of perforation of the small intestine caused by ingested foreign bodies", Eur Radiol, 14(10). 1918-1925. October 2004.

[10] Ambe, P., Weber, S.A., Schauer, $M$ and Knoefel, W.T, "Swallowed foreign bodies in adults", Dtsch Arztebl Int, 109(50). 869-875. December 2012.

[11] Dray, X. and Cattan, P, "Foreign bodies and caustic lesions", Best Pract Res Clin Gastroenterol, 27(5). 679-689. October 2013.

(C) The Author(s) 2019. This article is an open access article distributed under the terms and conditions of the Creative Commons Attribution (CC BY) license (http://creativecommons.org/licenses/by/4.0/). 